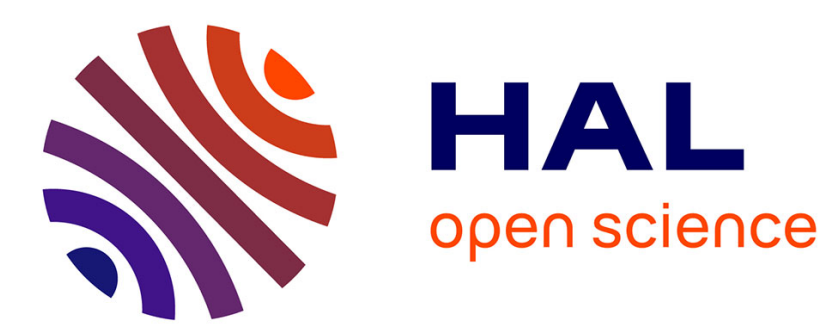

\title{
Probing living cells permeabilization dynamics by terahertz attenuated total reflection \\ Guilhem Gallot
}

\section{To cite this version:}

Guilhem Gallot. Probing living cells permeabilization dynamics by terahertz attenuated total reflection. SPIE Optical Engineering + Applications, SPIE, 2021, San Diego, United States. pp.118270S, 10.1117/12.2596166 . hal-03414068

\section{HAL Id: hal-03414068 \\ https://hal.science/hal-03414068}

Submitted on 4 Nov 2021

HAL is a multi-disciplinary open access archive for the deposit and dissemination of scientific research documents, whether they are published or not. The documents may come from teaching and research institutions in France or abroad, or from public or private research centers.
L'archive ouverte pluridisciplinaire HAL, est destinée au dépôt et à la diffusion de documents scientifiques de niveau recherche, publiés ou non, émanant des établissements d'enseignement et de recherche français ou étrangers, des laboratoires publics ou privés. 


\section{Probing living cells permeabilization dynamics by terahertz attenuated total reflection}

Gallot, G.

G. Gallot, "Probing living cells permeabilization dynamics by terahertz attenuated total reflection," Proc. SPIE 11827, Terahertz Emitters, Receivers, and Applications XII, 118270S (1 August 2021); doi: 10.1117/12.2596166

EDIE Event: SPIE Optical Engineering + Applications, 2021, San Diego, California, United States 


\title{
Probing living cells permeabilization dynamics by terahertz attenuated total reflection
}

\author{
G. Gallot ${ }^{\mathrm{a}}$

 \\ 91128 Palaiseau, France
}

\begin{abstract}
Using attenuated total reflection (ATR) in the terahertz domain, we demonstrate non-invasive, non-staining real time measurements of cytoplasm leakage during permeabilization of live epithelial cells by saponin detergent and after electropermeabilization. The origin of the contrast observed between cells and culture medium is addressed by both experimental and theoretical approaches, and gives access to permeabilization dynamics of live cells in real time. We show that terahertz modalities are more sensitive than fluorescence microscopy which is the reference optical technique for electropermeabilization. We propose analytical models for the influx and efflux of non-permeant molecules through permeabilized cell membranes.
\end{abstract}

Keywords: Terahertz, ATR, cell membrane permeabilization, detergent, electropermeabilization

\section{INTRODUCTION}

Reversible permeabilization of live cells is a complex and increasingly addressed issue, whether it is for medical application, or in lab research protocols where a constant effort is made to reach more realistic investigation conditions in biological systems. It is characterized by increased molecule transfer through the cell membrane. Applications cover anticancer drugs or imaging markers delivery, gene therapy, etc. Reversible permeabilization is mostly obtained by techniques creating pores into the membrane, the most commons being electroporation, non-ionic detergents and pore-forming toxins. ${ }^{1,2}$

The terahertz region has been shown to have potential in biomedical applications, but strong experimental limitations had long kept the study of biological objects down to the single purified molecule, simplified and/or pre-treated biological structures. Recent works demonstrated the possibility to address more complex systems, such as cells and even accessible tissues or small organs. ${ }^{3-6}$


Figure 1. THz-ATR device inserted in the THz-TDS setup. (A) The sample is located on top of a silicon support put in close contact with the top of the silicon prism. The support can be translated. Polarization is in the incident plane (p-polarization). (B) Close view of the ATR prism. The cell layer lyes in the evancescent field zone (in color gradient).

Terahertz Emitters, Receivers, and Applications XII, edited by Manijeh Razeghi,

Alexei N. Baranov, Proc. of SPIE Vol. 11827, 118270S - (c) 2021 SPIE

CCC code: $0277-786 X / 21 / \$ 21 \cdot$ doi: $10.1117 / 12.2596166$ 


\section{TERAHERTZ ATR MEASUREMENTS}

We developed attenuated total reflection (ATR) devices designed for biological samples (figure 1A), demonstrating the follow up of permeabilization dynamics of live cells in real time, without any fluorescent marker or complex preparation. ${ }^{5,7}$

The principle is as follows: the terahertz radiation directly measures the optical dielectric constant $\epsilon$ of the biological liquids inside or outside the cells. This $\epsilon$ is the sum of three components: ${ }^{8}$ the one of the core of the solute (from small peptides to big proteins), the one of the solvation shell (water molecules influenced by the solute) and the one of bulk water (figure 2). Since the total $\epsilon$ is directly related to the solute concentration, the measurements provide the concentration of the solutes. In order to probe cellular layers with a typical thickness of $10 \mu \mathrm{m}$, we designed ATR devices ${ }^{7}$ taking advantage of the existence of a thin evanescent wave, whose thickness $\delta$ is proportional and much less than the wavelength (figure 1B). An effective measurement then requires that $\delta$ be of the same magnitude as the cell thickness. At $1 \mathrm{THz}, \delta$ is about $20 \mu \mathrm{m}$, and strongly decreases in the infrared far below cell thickness, while it is too large in the microwave domain. The terahertz domain is then perfectly adapted to the study of cytosol concentration dynamics such as those resulting from cell membrane perturbations, but it is still very demanding in terms of technology. We performed our experiments using timedomain systems and femtosecond lasers to generate and detect the terahertz radiation, which results in very complicated and costly systems. However, new promising technologies are arising from research laboratories, such as the quantum cascade lasers.

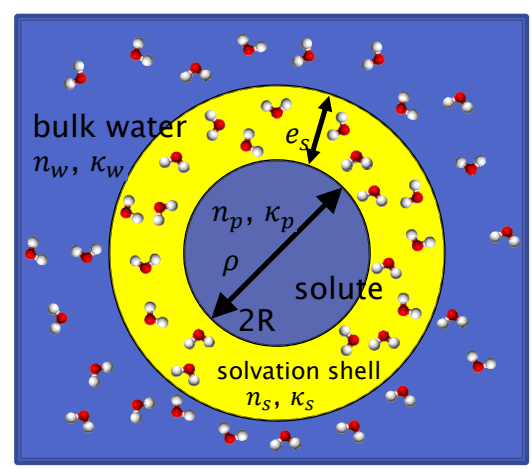

Figure 2. Solvation shell around solutes probed by terahertz radiation.

A 10- $\mu$ m-thick layer of epithelial MDCK (Madine-Darby Canine Kidney) cells was grown on a high resistivity silicon window, and then put on top of a silicon ATR prism. ${ }^{5}$ The evanescent field resulting from internal reflection occurs at the surface of the window, and probes the epithelial cell mono-layer. No staining nor any sample preparation are needed in this non-invasive imaging device.

\section{CELL MEMBRANE PERMEABILIZATION}

The plasma membrane can be disrupted by physical means such as electroporation, mechanical force, optoporation, thermal effect, biochemical techniques, etc. In the following, the permeabilization of MDCK epithelial cell membranes was artificially triggered by chemical (detergent) and physical means (electropermeabilization).

\subsection{Permeabilization by electropermeabilization}

The permeabilization of the live cells membrane by the delivery of electric pulses has fundamental interest in medicine, in particular in tumors treatment by electrochemotherapy. Since underlying mechanisms are still not fully understood, we studied the impact of electric pulses by terahertz ATR (Fig.3 A) and compared with fluorescence measurements. ${ }^{9}$ A dose effect was observed after cells exposure to different field intensities and a major

Further author information: (Send correspondence to G.G.)

G.G.: E-mail: Guilhem.Gallot@polytechnique.edu. 

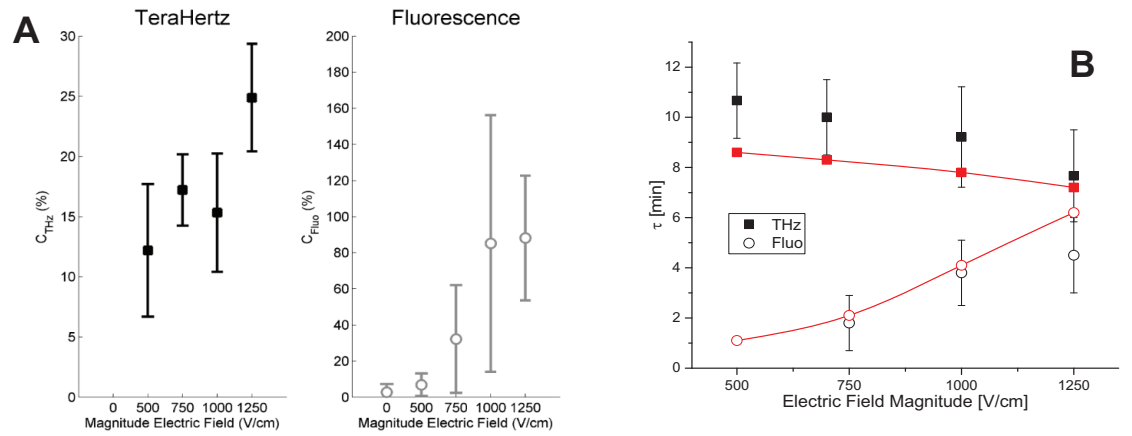

Figure 3. Evolution of the exponential decay time and concentration variation of the $\mathrm{THz}$ and fluorescence measurements of live MDCK cells exposed to electric pulses with different electric field magnitudes. A) Plateau values $\mathrm{C}_{\mathrm{THz}}$ and $\mathrm{C}_{\mathrm{fluo}}$ for the THz and Yo-Pro-1 fluorescence normalized signals. B) Time constants for the THz (squares) and Yo-Pro-1 fluorescence (circles) normalized signals. Data in red correspond to the values obtained with the theoretical model. The electric pulse parameters were fixed to 8 pulses, $100 \mu$ s and $1 \mathrm{~Hz}$.

impact on cell peptide/protein content was found (Fig.3 B). THz measurements suggest a leakage of proteins and other intracellular compounds. We show that terahertz modalities are more sensitive than fluorescence microscopy which is the reference optical technique in the case of electropermeabilization. We propose a new analytical model for the influx and efflux of non-permeant molecules through transiently (electro)permeabilized cell membranes (Fig.3 B).

\subsection{Permeabilization by detergent}

The saponins are glycosides mainly produced by plants that form soap-like foams in solution. Saponins consist of a sugar moeity linked to a hydrophobic aglycone. All the experiments were done with the same saponin sample to preserve the direct comparison between the experiments. The saponin mass concentration is referred as $\gamma_{0}$.

Terahertz Attenuated Total Reflection (THz-ATR) makes use of the evanescent wave at the back of a prism under total internal reflection, which is coupled to the sample under study (Fig. 1). If an absorbing layer such as cells is put on top of the prism, it couples with the evanescent field and decreases the amount of reflected energy. The measurement of the reflected beam is then directly correlated with the dielectric constant of the medium topping the prism. More precisely, we consider a thin layer of cells and the surrounding solution in this inhomogeneous evanescent wave. Providing that the thickness of the cell layer matches the penetration depth of the evanescent wave, the reflected terahertz wave is modified by the terahertz dielectric properties of the cell layer in contact with the top of the prism. The stronger coupling is obtained for a penetration depth of the same value as the cell layer thickness. The resulting reflected terahertz signal is then correlated with the cytosol content. $^{7,8}$

For the modeling, the system is considered to be a uniform cell layer. The basal plasma membrane is in contact with the silicon substrate while the apical membrane is in direct contact with saponin molecules in solution in PBS. The saponin molecules can move freely in the liquid by diffusion or sedimentation. Once a molecule encounters the apical membrane it creates a pore with a given probability $\eta$, increasing the total number of pores with time. Then, effusion of molecules through the pores takes place and changes the concentration of molecules in cytosol. The saponin concentration of the solution is not considered to be modified since the PBS/saponin solution volume is by far much larger than that of cytosol. Pores are allowed to grow or shrink with a characteristic exponential time $\tau$. One obtains the analytical solution for the evolution of the normalized terahertz signal

$$
S(t)=\exp \left\{-\varphi \tau^{3 / 2}\left[\frac{\sqrt{\pi}}{2} \exp \left(\frac{t}{\tau}\right) \operatorname{erf} \sqrt{\frac{t}{\tau}}-\sqrt{\frac{t}{\tau}}\right]\right\} \quad \text { with } \quad \varphi=\frac{2 \eta \mathcal{N}_{A} \gamma_{0} S_{p 0} D_{c} \sqrt{D}}{\sqrt{\pi} L \delta M}
$$




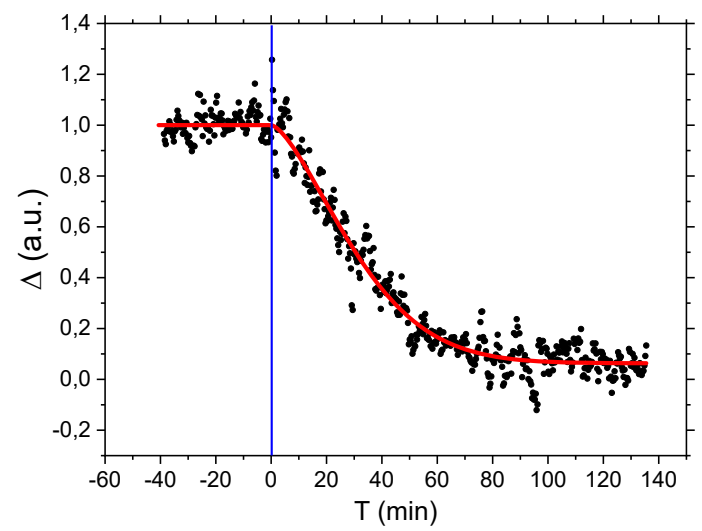

Figure 4. THz-ATR signal $\Delta(t)$ for MDCK cell layer after addition of saponins at $T=0$ for experimental data (black dots) and best fit (red line). The blue vertical line shows addition of saponin.

where $\tau$ is the characteristic time for the pore evolution, $D$ and $D_{c}$ are saponin and cytosol diffusion constants, $S_{p 0}$ is the initial pore surface, $L$ is the cell thickness, $\delta$ is the membrane thickness, $M$ the saponin molar mass and $\eta$ the efficiency of the binding of a saponin molecule to the cell membrane. An example of experimental setup is given in Figure 4 with the best fit from Eq. 1.

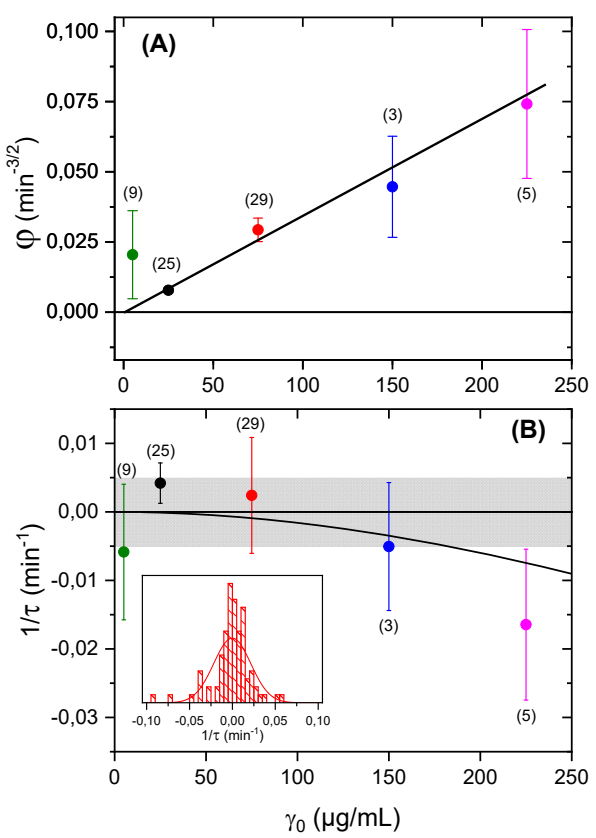

Figure 5. Model parameters $\varphi(\mathbf{A})$ and $1 / \tau(\mathbf{B})$ versus saponin mass concentration $\gamma_{0}$. (A) The line is the best fit proportional function. (B) The grey rectangle is a guide for the eye. The black line is the apparent $1 / \tau$ evolution considering overlapping of the pores. Inset shows the distribution of $1 / \tau$ for the whole concentrations and a Gaussian fit. For both, the number of experiments are shown in parenthesis.

We experimented the effect of the initial concentration of saponins on the membrane permeability dynamics probed by THz-ATR. ${ }^{10}$ Experiments refer to time $T$ following a perturbation applied to the cells at $T=0$. We record the THz-ATR signal $\Delta$ for 30 min to make sure the signal is stable. Then, at $T=0$ we add $30 \mu \mathrm{L}$ of 100X-concentrated solution of saponins in PBS with a micropipette so that the resulting saponins concentration equals $\gamma_{0}$. With least square regression, the experimental data are fitted to provide the parameters $\tau$ and $\varphi$. First, for the cell geometry and diffusion parameter $\varphi$, a linear dependency versus $\gamma_{0}$ is experimentally observed, as expected from Eqs. 1. Second, we studied the variation of the pore size evolution characteristic time (see 
Fig. 5B). At first sight, $1 / \tau$ is very close to 0 within experimental uncertainty, shown in grey in Fig. $5 \mathrm{~B}$. This corresponds to $|1 / \tau|<0.05 \mathrm{~min}^{-1}$ and then to $|\tau|>200 \mathrm{~min}$. This means that pores created by saponins exhibit a very slow dynamics if any, longer then $200 \mathrm{~min}$ in any case. Furthermore, Fig. 5B seems to exhibit a small discrepancy with respect to purely static pores, for larger saponins concentration. The experimental values of $1 / \tau$ are slightly negative for high saponins concentrations which would evoke a large but not infinite decaying pore size time. However, this appears to be an artifact due to pore overlapping, as shown by the black line, which is the apparent $1 / \tau$ evolution considering pore overlapping. Taking into account overlapping, we found that two effects are opposing during permeabilization: the pore overlapping related to saponin molecules diffusion, and the efflux dynamics of cytosol molecules.

We found a good correlation between the experimental data and the model. In particular, we found that the pores created by saponin molecules appear to be static within experimental uncertainty, and that the probability for a saponin molecule to bind to the membrane is found to be very efficient, with a probability greater than $50 \%$. Therefore, the most limiting parameter in the MDCK permeabilization by saponin molecules is the diffusion of saponin molecules.

\section{REFERENCES}

[1] Hapala, I., "Breaking the barrier: Methods for reversible permeabilization of cellular membranes," Crit. Rev. in Biotechn. 17, 105-122 (1997).

[2] Wassler, M., Jonasson, I., Persson, R., and Fries, E., "Differential permeabilization of membranes by saponin treatment of isolated rat hepatocytes," Biochem. J 247, 407 (1987).

[3] Masson, J. B., Sauviat, M. P., Martin, J. L., and Gallot, G., "Ionic contrast terahertz near field imaging of axonal water fluxes," Proc Natl Acad Sci USA 103(13), 4808-4812 (2006).

[4] Masson, J.-B., Sauviat, M.-P., and Gallot, G., "Ionic contrast terahertz time resolved imaging of frog auricular heart muscle electrical activity," Appl. Phys. Lett. 89, 153904 (2006).

[5] Wojdyla, A. and Gallot, G., "Attenuated internal reflection terahertz imaging," Opt. Lett. 38, 112-114 (2013).

[6] Shiraga, K., Ogawa, Y., Suzuki, T., Kondo, N., Irisawa, A., and Imamura, M., "Characterization of dielectric responses of human cancer cells in the terahertz region," Journal of Infrared Millimeter and Terahertz Waves 35(5), 493-502 (2014).

[7] Grognot, M. and Gallot, G., "Quantitative measurement of permeabilization of living cells by terahertz attenuated total reflection," Appl. Phys. Lett. 107, 103702 (2015).

[8] Grognot, M. and Gallot, G., "Relative contributions of core protein and solvation shell in the terahertz dielectric properties of protein solutions," J. Phys. Chem. B 121(41), 9508-9512 (2017).

[9] Azan, A., Grognot, M., García-Sánchez, T., Descamps, L., Untereiner, V., Piot, O., Gallot, G., and Mir, L. M., "Monitoring the molecular composition of live cells exposed to electric pulses via label-free optical methods," Scientific reports 10, 10471 (2020).

[10] Zheng, X. and Gallot, G., "Dynamics of cell membrane permeabilization by saponins using terahertz attenuated total reflection," Biophysical J. 119, 749-755 (2020). 